\title{
Strengths and limitations of a musculoskeletal model for an analysis of simulated meat cutting tasks
}

\author{
Charles Pontonnier $^{\mathrm{a}, \mathrm{b}, \mathrm{c}}$, Mark de Zee ${ }^{\mathrm{a}}$, Afshin Samani ${ }^{\mathrm{a}}$, Georges Dumont ${ }^{\mathrm{b}, \mathrm{c}}$, Pascal Madeleine ${ }^{\mathrm{a},{ }^{*}}$ \\ a. Center for Sensory-Motor Interaction (SMI), Department of Health Science and Technology, \\ Aalborg University, Fredrik Bajers Vej 7, Bldg. D-3, DK 9220 Aalborg, Denmark \\ b. MimeTIC, IRISA-INRIA Rennes, Campus de Beaulieu, 35042 Rennes Cédex, France \\ c. Département mécatronique, ENS Cachan Antenne de Bretagne, 35170 Bruz Cédex, France
}

\section{Corresponding author:}

Prof. P. Madeleine, Ph.D., dr.scient., Physical Activity and Human Performance group, Center for Sensory-Motor Interaction (SMI), Dept. of Health Science and Technology, Aalborg University, Fredrik Bajers Vej 7, 9220 Aalborg East, Denmark; e-mail: pm@hst.aau.dk

\begin{abstract}
This study assessed the capacity of a musculoskeletal model to predict the relative muscle activation changes as a function of the workbench height and the movement direction during a simulated meat cutting task. Seven subjects performed a cutting task alternating two cutting directions for 20s at four different workbench heights. Kinematics, electromyography (EMG), and cutting force data were collected and used to drive a musculoskeletal model of the shoulder girdle. The model predicted the muscle forces exerted during the task. Both the recorded and computed activation of the muscles was then compared by means of cross-correlation and by comparison of muscle activation trends with respect to the workstation parameters, i.e. cutting direction and work bench height. The results indicated that cutting movements involving arm flexion are preferable to movement requiring internal arm rotation and abduction. The optimal bench height for meat cutting tasks should be between 20 and $30 \mathrm{~cm}$ below the worker's elbow height. The present study underlines a beneficial use of musculoskeletal models for adjusting workstation parameters.
\end{abstract}

Keywords - Electromyography, motion capture, trend analysis, muscle forces, physical risk factors 


\section{Introduction}

The last European Working Condition Survey reports an exposure of $63 \%$ of the working population to repetitive hand or arm movement, and $46 \%$ of the population mentions painful, tiring positions for at least one quarter of the working time (Fifth European Working Conditions Survey, 2010). Indeed, the prevalence of occupations involving physical labour has not changed over the last 20 years in Europe. The risk factors implicated in the development of work-related musculoskeletal disorders (WMSD) are commonly divided into internal and external risk factors (Sjøgaard et al., 1995). Individual factors like age, gender, fitness level, and personality are known as internal risk factors. The external risk factors are expressed in terms of physical and psychosocial components. A relatively fixed erect posture, repetitive arm movements, heavy work, insufficient rest, vibrations as well as static posture are recognised important physical factors contributing to WMSD (Winkel and Westgaard, 1992; Sjøgaard et al., 1995), which are often reported in the neck-shoulder of butchers (OSHA, 2007). Stress and pain behaviour have also been identified as some of the important psychological risk factors. Thus, the presence of internal and external risk factors emphasizes the complex aetiology of WMSD. Further, the inter-relationships among the risk factors do not facilitate the evaluation of the impact of ergonomic interventions.

Musculoskeletal simulation enables to address this issue as the load over a region of the musculoskeletal system can be evaluated during an ergonomic intervention aiming at reducing the occurrence of WMSD. Musculoskeletal simulation is of particular interest when designing a workstation. Such approach is cost effective as it enables to address most of the physical risk factors without the need for human testing prior to the prototype stage. Moreover, musculoskeletal simulation processes a large number of biomechanical parameters (kinematics, kinetics, muscle forces) for the whole model. In comparison, bipolar surface electromyography (EMG) recordings provide only temporal aspects of the level of activation from the muscle of interest. However, EMG recording are still often performed in ergonomics for such purpose (Madeleine et al., 2011). Thus, musculoskeletal simulation offers undeniable advantages when evaluating the design of a workstation. Finally, the fitting of the workstation to the worker can be done easily and may in the end result in a gain of time and money (Weidlich et al., 2009).

A pre-requisite for using musculoskeletal models for the workplace evaluations is that the predictions of the internal loads are correct. The validations of musculoskeletal simulations are often made by comparison of EMG signals recorded during the task; see for example de Zee et al. (2007). The qualitative validation is based on comparison of the shape of the recorded and computed activation patterns (Erdemir et al., 2007). In ergonomics, a quantitative evaluation of the internal loads is crucial when the design parameters are changed. A quantitative validation of the loads predicted by the musculoskeletal model can be done with objective criterions such as the difference between the work performed by internal and external forces or curve comparison metrics in magnitude (Lund et al., 2012). The validation can also be done by comparing the trends followed by the computed and recorded activation of the muscles as a function of design parameters (Pontonnier et al., 2011). This latter approach is of particular interest as the trends followed by the muscle activation provide direction to optimize the design of the work station. To the best of our knowledge, quantitative validation by trend analysis is still rarely used in ergonomics.

The present study aimed at filling this gap by validating a musculoskeletal model of the upper limb for standardized meat cutting task. Validation of such models makes it possible to provide ergonomic guidelines in a work station design process. We opted for a simulated meat cutting task similar to the one presented by Madeleine et al. (1999) to test a musculoskeletal model in relation to work place design. For that purpose we analyzed and compared the trends followed by computed and recorded activation of the muscles during repetitive arm movement as a function of change in cutting direction and in the height of the workbench. The trend analysis of the recorded activations (obtained from 
EMG data) has been previously reported (Pontonnier et al. 2012), but not compared with the computed activations obtained from the musculoskeletal model.

\section{Materials and methods}

\subsection{Experimental protocol}

Seven male subjects (mean age $\pm s d$ : $29 \pm 5$ years old, height: $182 \pm 8 \mathrm{~cm}$, weight: $77 \pm 16 \mathrm{~kg}$ ) participated in the present study. Informed consent was obtained from the subjects prior to the recordings. All participants reported no pain in the shoulder region prior to the experiment and had no history of neck-shoulder disorders. The study was approved by the local ethics committee (No. N-20070004) and conducted in accordance with the Declaration of Helsinki.

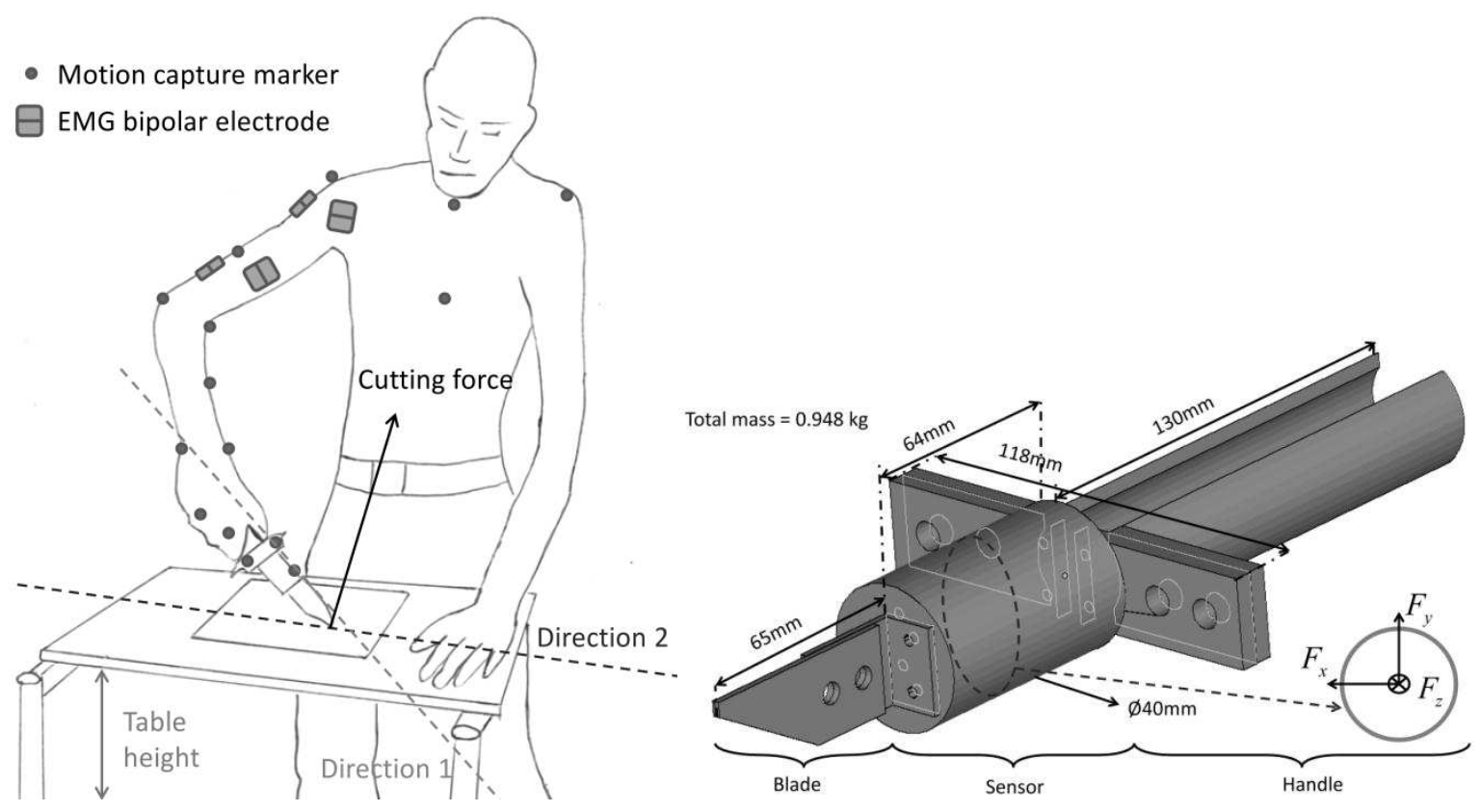

Figure 1: Experimental situation showing the instrumented knife, bipolar EMG electrodes, and the active markers for the assessments of the cutting forces and movement in 3D. Motion capture markers were placed on standard anatomical landmarks. The resulting cutting force was measured mainly in the $(y, z)$ plane, and lateral bending effects appeared in the $x$ direction.

An illustration of the experimental set-up is depicted in figure 1 . The subjects were standing in front of a workbench. The workbench height was set at 4 different heights: $0,-10,-20$, and $-30 \mathrm{~cm}$. The 0 $\mathrm{cm}$ height corresponded to the subject's elbow height and is the recommended height for light work (McCormik and Sanders, 1987). Two main directions of cutting were used, i.e.: 1) arm flexion, and 2) a combination of internal arm rotation and abduction. These directions were chosen to simulate real slaughterhouse work situations observed in the meat industry (Madeleine et al., 1999). The subjects were asked to perform cutting tasks in these two directions for $20 \mathrm{~s}$ at each workbench height. The cycle combined a static postural phase (holding), a cutting phase in direction 1 (diagonal slot), and a cutting phase in direction 2 (horizontal slot). The recordings consisted of 4 sequences over $20 \mathrm{~s}$ in each trial. A practice period was performed to teach the subjects to apply a resultant force of approx. $50 \mathrm{~N}$ in agreement with the forces measured during a real meat cutting task (Juul-Kristensen et al., 2002; McGorry et al., 2003). 


\subsection{Data acquisition}

The collected data consisted of cutting forces, kinematics data, and surface EMG data. A trigger was used to synchronize the motion capture, cutting force, and EMG acquisition, using an End-of-Frame signal starting from the motion capture markers.

\subsubsection{Cutting force acquisition}

The main part of the literature analyzing the cutting force exerted during meat cutting is based on relatively simple force assessment as only a one-dimensional force was measured (Madeleine et al., 1999; Juul-Kristensen et al., 2002). However, assessing the cutting forces in 3D exerted by the worker offers advantages. McGorry (2001) has designed an advanced knife instrumented with strain gauges to record reactive forces and grip moments. In our study, a 3D force registration was necessary to perform a 3D inverse dynamics analysis of the task. Thus, 3D cutting forces were recorded using a newly designed 3D instrumented knife. The design of the knife is based on the instrumentation of a 3D force sensor (FS6-2000, AMTI, Watertown, MA, USA). The reaction forces applied to the transducer were recorded (figure 1). The blade had the shape of a real knife considering that only the tip of the blade is used during a cutting event. The blade thickness avoided bending issues during the cutting task. The knife had to be handled as a real knife, i.e. the handle diameter was similar to knives used in the Danish slaughterhouses. A tennis grip was added to facilitate grasping. The knife was approximately $0.5 \mathrm{~kg}$ heavier than knives used in Danish slaughterhouses. The mass difference was due to the use of the 3D force sensor and was taken into account in the simulation (see below). Force signals were low-pass filtered $(10.5 \mathrm{~Hz})$ and amplified 2000 times. The signals were A/D converted and sampled at $60 \mathrm{~Hz}$ (12 bits A/D converter, Nidaq 6024, National Instruments, Austin, TX, USA) and recorded through a custom made program in LabView 8.2 (National Instruments, Austin, TX,USA), which also provided feedback on the applied forces to the experimenter.

\subsubsection{Motion acquisition}

The motion was captured using an active marker motion capture system (Visualeyez $\mathrm{II}^{\mathrm{TM}}$ system set up with two VZ4000 trackers, Phoenix Technologies Inc., BC, Canada). Sixteen active markers were used to collect trunk, right arm, and knife motion. Kinematics data were sampled at $60 \mathrm{~Hz}$. The missing markers were interpolated using a rigid body hypothesis. Finally, 3D coordinates were lowpass filtered $(5 \mathrm{~Hz})$.

\subsubsection{Surface EMG acquisition}

Four bipolar channels were used to collect EMG from the deltoideus medialis, deltoideus anterior, biceps brachii, and triceps long head (figure 1) with bipolar surface electrodes (Neuroline 720, Ambu, Denmark). A reference electrode was placed on the $\mathrm{C} 7$ spinal process. The bipolar surface electrodes were aligned (inter-electrodes distance: $2 \mathrm{~cm}$ ) on abraded ethanol-cleaned skin along the direction of the muscle fibers. The bipolar electrodes were placed with respect to anatomical landmarks following the recommendations (SENIAM, 1999). The EMG signals were amplified 2000 times (128channel surface EMG amplifier, LISiN-OT Bioelectronica, Torino, Italy) and sampled at $2048 \mathrm{~Hz}$. The signals were full-wave rectified and low pass filtered $(4 \mathrm{~Hz})$ to obtain the absolute linear envelope of the muscle activation profiles.

\subsection{Inverse dynamics analysis}

The collected data were processed to drive a detailed musculoskeletal model based on inverse dynamics of the trunk and the right arm similar to the one developed by Rasmussen and de Zee (2010). The model was built in the AnyBody Modeling System (AnyBody Technology A/S, Aalborg, Denmark). The principles and the mathematical details behind the software are described in Damsgaard et al. (2006). The musculoskeletal model has 52 degrees of freedom, 135 muscle 
actuators, and is based on the data by Van der Helm (1994). The model is available at the AnyBody Managed Model Repository ${ }^{1}$. The musculoskeletal model is scaled to fit the morphology of the subject, and marker positions are optimized to fit real landmarks using an optimization routine described by Andersen et al. (2010). The 3D cutting force was then used to define the external forces applied to the model, and mass and inertia properties of the knife were taken into account in the simulation. Ten $\mathrm{s}$ of each trial were simulated, discarding the first and last $5 \mathrm{~s}$.

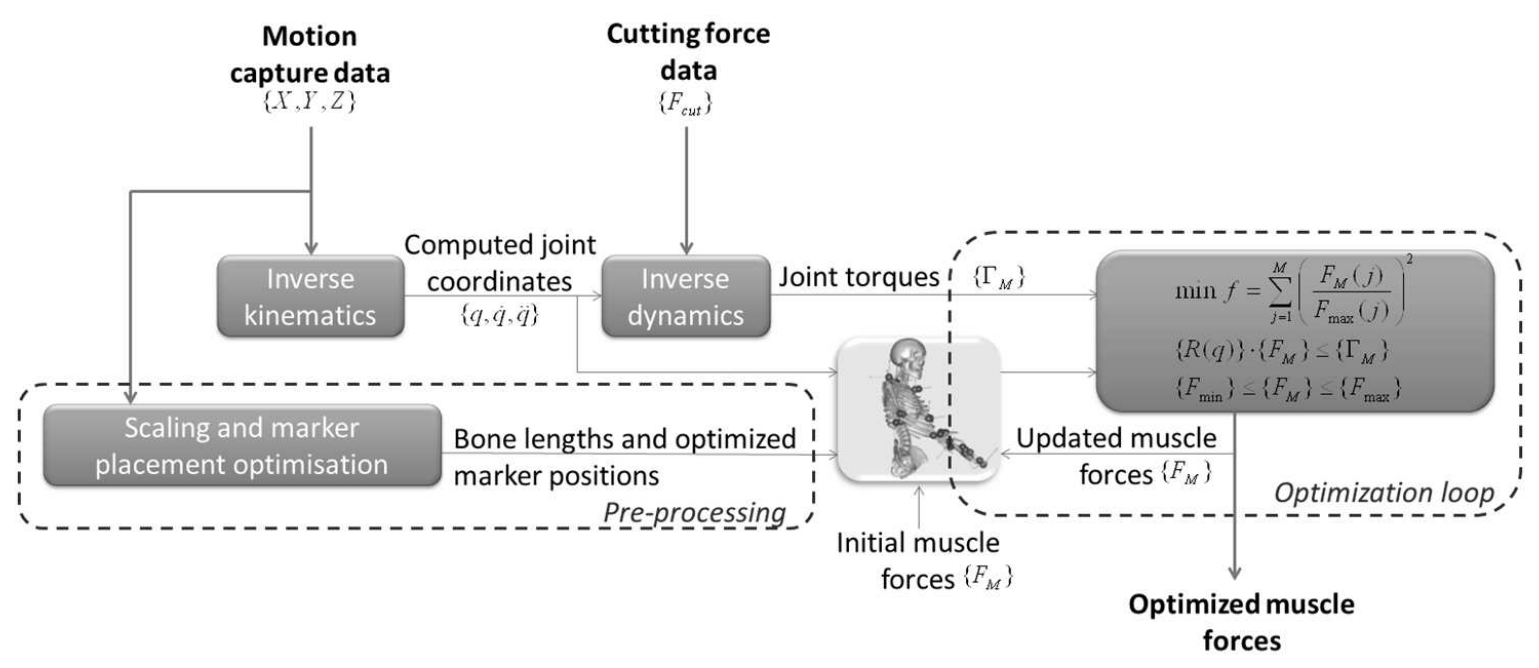

Figure 2: Inverse dynamics pipeline for muscle forces estimation.

An inverse dynamics method was used to generate the muscle forces from kinematics (figure 2). The objective function used for the muscle recruitment step was a standard quadratic criterion:

$$
\min f=\sum_{j=1}^{n}\left(\frac{F_{j}(t)}{F_{j}^{\max }}\right)^{2}
$$

Where $F_{j}(t)$ is the current muscle force of muscle $\mathrm{j}$ at the time $\mathrm{t}$, and $F_{j}^{\max }$ the maximum isometric force that muscle $\mathrm{j}$ can produce. We used the maximum isometric forces reported in Van der Helm (1994). The muscles are described as simple actuators as shown in this equation:

$$
F_{j}(t)=a_{j}(t) \cdot F_{j}^{\max }
$$

Where $\mathrm{a}_{j}(\mathrm{t})$ is the muscle activation with 0 corresponding to no activation and 1 to maximal activation.

\subsection{Validation processing \& statistics}

The validation process consisted of qualitative and quantitative comparisons of recorded and computed activation of the muscles. The ranges of motion were also computed to assess intersubject variability. Figure 3 shows the global processing scheme used to validate the simulation.

Qualitative comparison of the recorded and computed activation of the muscles was performed by computing a normalized cross-correlation between both the surface EMG signals and the corresponding estimated force trajectories (computed). The cross-correlation function is a measure of similarity of two waveforms as a function of a time-lag applied to one of these. Between recorded

\footnotetext{
${ }^{1}$ http://forge.anyscript.org/gf/project/ammr/
} 
and computed activation, contraction and activation dynamics of muscles were considered to introduce a positive time-lag less than $200 \mathrm{~ms}$ - global contraction and activation dynamics delay (Cavanagh and Komi, 1979; van Dieën et al., 1999). Therefore, the highest level of correlation obtained from the cross-correlation in a $[0,200 \mathrm{~ms}]$ positive time-lag window was considered as the real correlation between recorded and computed signals. The cross-correlation was first applied to the entire simulation sequence $(10 \mathrm{~s})$. The second time, the trials were divided into two phases: static postural phases, corresponding to an exerted force level equal to the knife weight, and the dynamic phases consisting of cutting events in the two directions. During the cutting events, increases of the exerted force were detected (framed with two minimum points at the beginning and the end of a cutting event). Then a cross-correlation was computed locally for each phase to assess possible discrepancies of the model behavior with respect to the trial phases. The correlations between signals were obtained using the same time-lag as explained above (10 s sequence). Cross-correlation values were then averaged per subject and workbench height.

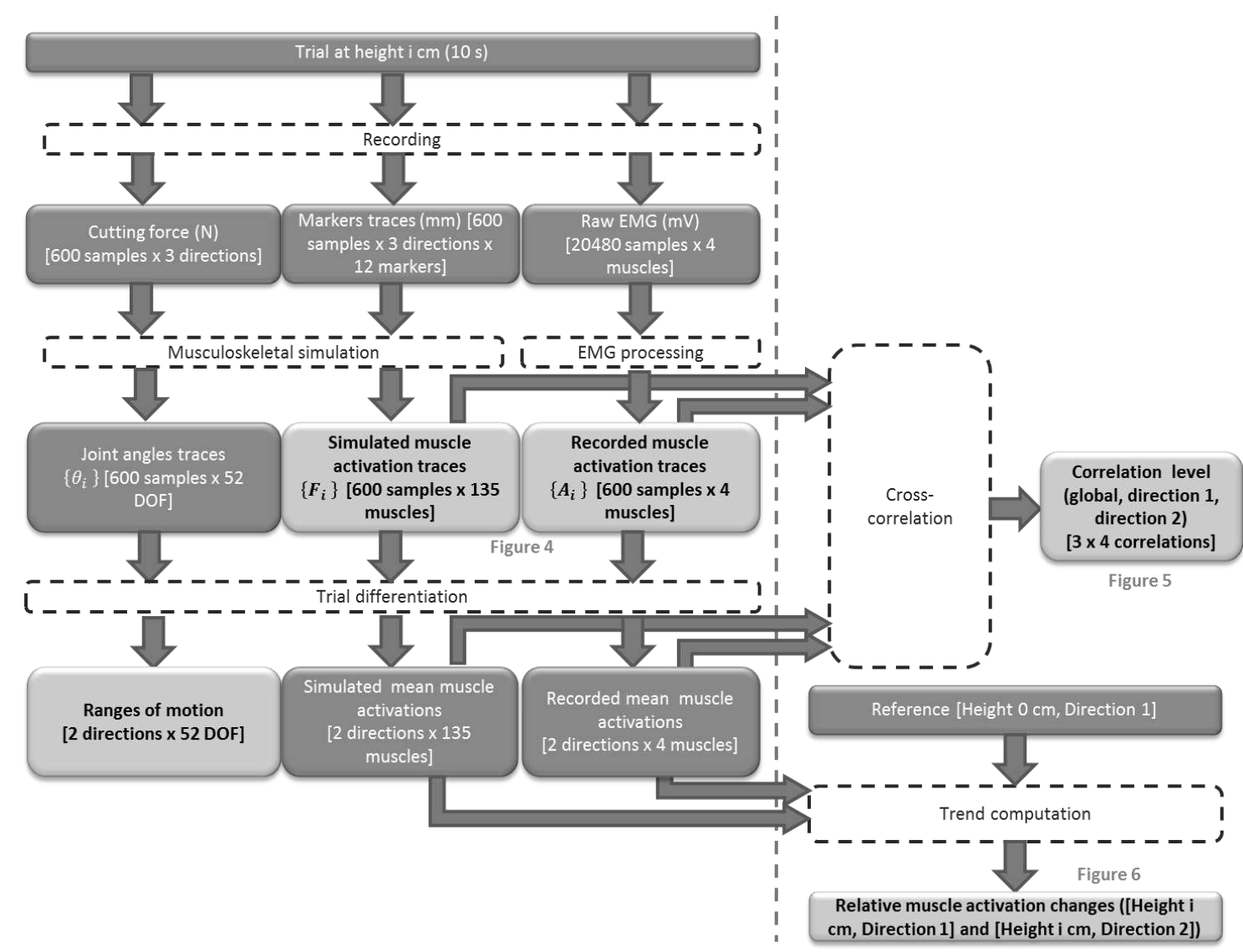

Figure 3: Global processing pipeline for data processing. The major outputs of the pipeline are highlighted in grey. Numbers in square brackets are the output dimensions. The outputs were averaged for the whole pool of subjects. The figure indications are referring to the other figures in the manuscript.

Further, to compare quantitatively computed and recorded activation of the muscles, a trend analysis was conducted associating the cutting phases and workbench heights. The trends computed for the recorded EMG have been previously reported in Pontonnier et al. (2012). In the current study, the trends were computed from the musculoskeletal simulation and then compared with the recorded trends. After division into phases, the recorded and computed activation of the muscles was averaged for each cutting event. Then, the mean+/-SD values corresponding to the two configurations ([Trial Height, Cutting direction 1] and [Trial Height, Cutting direction 2]) were computed for each trial. Thereafter, the averaged activation of the muscles was normalized for each 


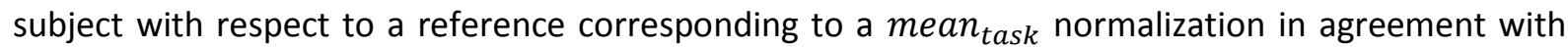
Burden (2010).The chosen reference in this study was $[0 \mathrm{~cm}$, direction 1]. The relative variations of activation of the muscles were computed as shown in equation 3 to account for the differences in terms of duration and exerted cutting forces between the trials.

$$
\left\{\begin{array}{c}
{ }_{j}^{i} R_{\text {com }}^{k}=\frac{{ }_{j}^{i} A_{\text {com }}^{k} \cdot{ }_{j}^{i} \alpha-{ }_{1}^{0} A_{\text {com }}^{k}}{{ }_{1}^{0} A_{\text {com }}^{k}} \\
{ }_{j}^{i} R_{\text {rec }}^{k}=\frac{{ }_{j}^{i} A_{\text {rec }}^{k} \cdot{ }_{j}^{i} \alpha-{ }_{1}^{0} A_{\text {rec }}^{k}}{{ }_{1}^{0} A_{\text {rec }}^{k}}
\end{array}\right.
$$

Where ${ }_{j}^{i} R_{c o m}^{k}$ and ${ }_{j}^{i} R_{\text {rec }}^{k}$ were the relative variations of the computed activation ${ }_{j}^{i} A_{c o m}^{k}$ and the recorded activation ${ }_{j}^{i} A_{\text {rec }}^{k}$ of muscle $\mathrm{k}$ in the [Height i, Direction j] configuration, respectively. The confidence factor ${ }_{j}^{i} \alpha$ allowed the assessment of differences in terms of cutting force and duration, and was computed as:

$$
{ }_{j}^{i} \alpha=\frac{{ }_{1}^{0} F \cdot{ }_{1}^{0} D}{{ }_{j}^{i} F \cdot{ }_{j}^{i} D}
$$

Where ${ }_{1}^{0} F$ was the mean exerted force, and ${ }_{1}^{0} D$ was the mean cutting task duration for the configuration [Height $0 \mathrm{~cm}$, Direction 1] (reference condition).

The relative variation was computed for each subject and averaged among subjects. Spearman's correlation coefficients were then calculated between series of recorded and computed relative variations for each subject and among subjects.

The range of motion (ROM) of elbow flexion, shoulder abduction, shoulder flexion, shoulder internal rotation, and wrist flexion during the cutting phases was extracted from the kinematics data. This provided information on the adopted motor control strategies. Finally, the resultant cutting force and cutting task duration were also computed. The ranges of motion, resultant cutting force, and cutting task duration were analyzed using a full factorial repeated measure analysis of variance with workbench height and cutting direction as independent factors. 

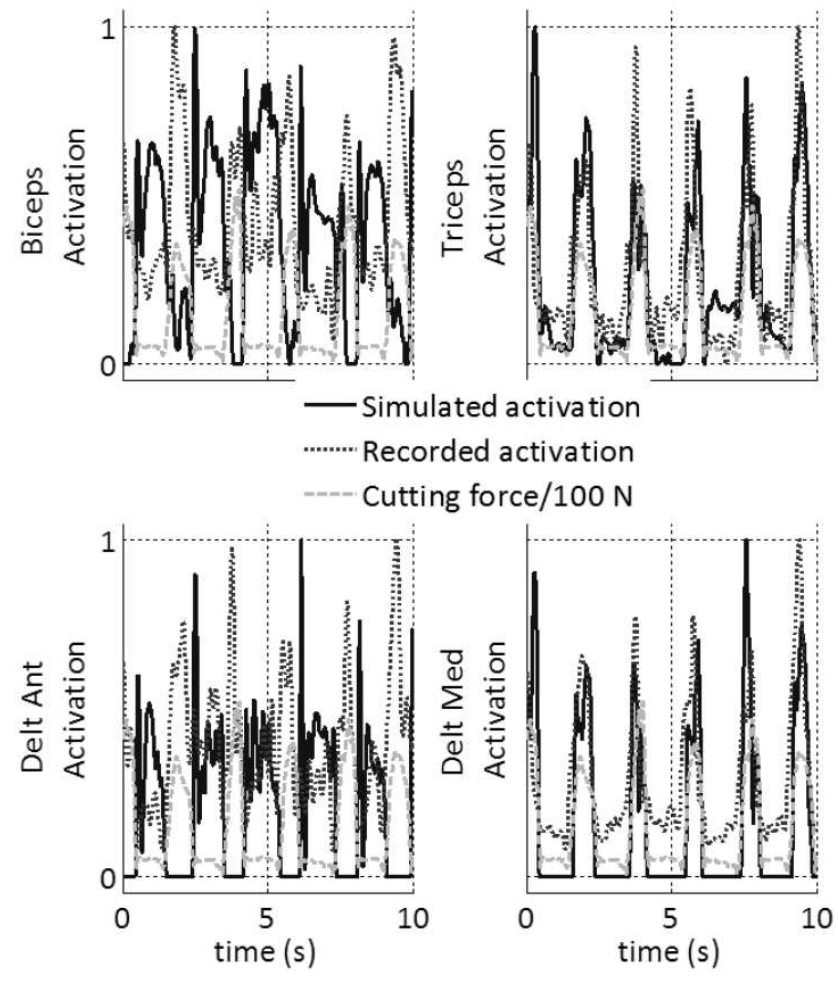

Figure 4: The results for a sample trial (subject 4, height $0 \mathrm{~cm}$ ). The resulting cutting force recorded via the knife is shown as a time reference (divided by 100). Recorded and computed activation of the muscles are normalized by the maximum reached during the trial to facilitate the comparison. Stimulation of $10 \mathrm{~s}$ is featured.

\section{Results}

\subsection{Overview}

Figure 4 shows the resultant cutting force and the recorded and computed activation of the muscles for subject 4 (workbench height: $0 \mathrm{~cm}$ ). The figure underlines the role of prime movers, i.e. triceps and deltoideus medialis muscles during cutting events. For these two muscles, both the recorded and computed activation of the muscles had similar patterns consisting of increases during cutting events and decreases to baseline values during postural phases of the work task. The biceps brachii and deltoideus anterior muscles exhibited more variable patterns, but followed in general the resultant cutting force. The recorded and computed EMG signals of triceps and deltoideus medialis were in phase and corresponded to the resulting force pattern of the muscle. 

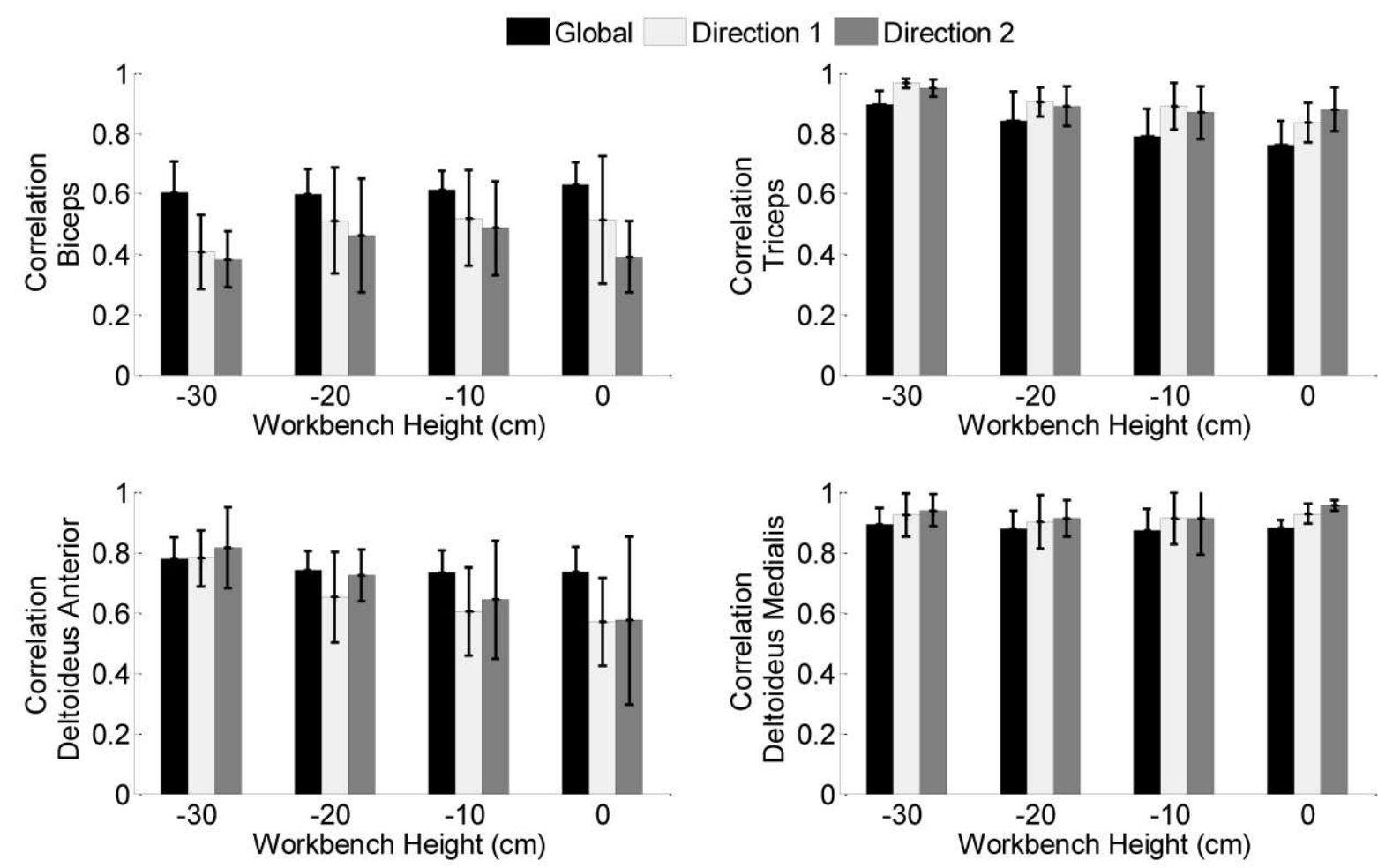

Figure 5: Mean correlation coefficients $\pm S D$ in relation to workbench height. Global represents the correlation for the entire trials, and direction 1 and 2 correspond respectively to the cutting event consisting of arm flexion and a combination of arm internal rotation and abduction.

Table 1: Mean cross-correlation coefficients \pm SD for the 7 subjects. Global represents the entire trial, direction 1 and 2 correspond to the cutting event consisting of arm flexion and a combination of arm internal rotation and abduction, respectively. ${ }^{*}$ emphasizes the highest results.

\begin{tabular}{|l|l|l|l|}
\hline Correlation & Global & Direction 1 & Direction 2 \\
\hline Biceps & $0.75 \pm 0.06$ & $0.65 \pm 0.08$ & $0.69 \pm 0.16$ \\
\hline Triceps & $\mathbf{0 . 8 8} \pm \mathbf{0 . 0 4 *}$ & $\mathbf{0 . 9 2} \pm 0.06^{*}$ & $\mathbf{0 . 9 3} \pm \mathbf{0 . 0 6 *}$ \\
\hline Deltoideus Anterior & $0.61 \pm 0.06$ & $0.49 \pm 0.13$ & $0.43 \pm 0.13$ \\
\hline Deltoideus Medialis & $\mathbf{0 . 8 2} \pm \mathbf{0 . 0 7 *}$ & $\mathbf{0 . 9 0} \pm \mathbf{0 . 0 4 *}$ & $\mathbf{0 . 8 9} \pm \mathbf{0 . 0 4 *}$ \\
\hline
\end{tabular}

\subsection{Signal correlation}

Figure 5 reports the averaged correlations at workbench heights of $0,-10,-20$, and $-30 \mathrm{~cm}$. Table 1 summarizes the cross-correlation results. The highest correlation coefficients were obtained for the deltoideus medialis and triceps muscles. The correlation coefficients were higher for triceps and deltoideus medialis muscles during the cutting events compared with values obtained over the entire trial (denoted Global on figure 5). The opposite pattern was seen for biceps brachii and deltoideus anterior. It implies that the computed activation of the muscles for biceps and deltoideus anterior corresponded better to the recorded activation during the static postural phases than during the cutting phases. Notably, subject 1 had a high correlation coefficient $(>0.7)$ during the cutting events 
in both cutting directions while the remaining subjects had a correlationcoefficient between 0.4 and 0.5 .
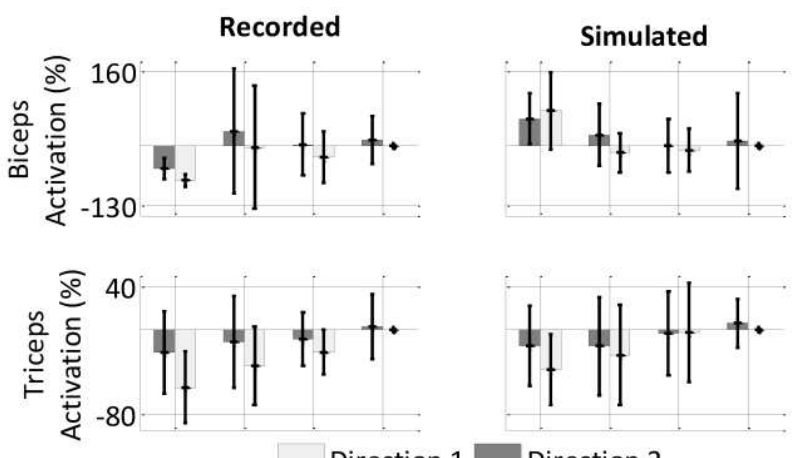

Direction 1 Direction 2
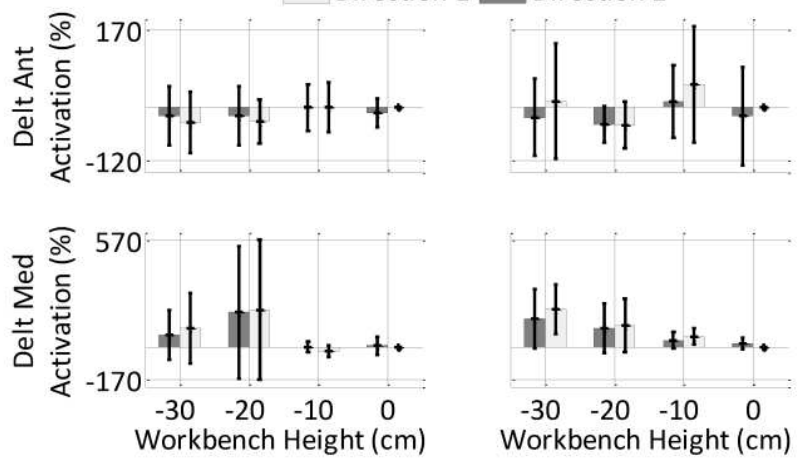

Figure 6: Relative activation levels with regard to directions 1 and 2 (Dir 1, Dir 2) and workbench height. Direction 1 and 2 correspond to the cutting event consisting of arm flexion and a combination of arm internal rotation and abduction, respectively. The left column shows recorded activation of the muscles and the right column the simulated ones.

\subsection{Trend comparison}

Figure 6 shows the recorded and the simulated trends in relation to the height of the workbench and the cutting direction. In general, the trend analysis confirms the results obtained from the signal correlation. The trends followed by simulated triceps and deltoideus medialis activation were relatively consistent with the recorded ones while this was seen to a lower extent for biceps and deltoideus anterior. The trend analysis provided similar information for both the recorded and the simulated muscle activation. Lower workbench height led to a decreased level of activation for triceps and an increased level of activation for deltoideus medialis. Direction 1 (diagonal slot) resulted in lower levels of activation. A slight difference appeared in the deltoideus medialis simulated trend since the simulation indicated that $-30 \mathrm{~cm}$ height resulted in lower activation level compared with $-20 \mathrm{~cm}$ while the recorded trends indicated the opposite.

Table 2: The overall correlations (Spearman) on muscle activation trends for all the subjects. Only significant correlation between the recorded and computed trends are reported ( $p<0.05)$. NS: Nonsignificant correlations.

\begin{tabular}{|l|l|l|l|l|}
\hline $\begin{array}{l}\text { Correlation on } \\
\text { activation trends }\end{array}$ & Biceps & Triceps & Deltoideus Anterior & Deltoideus Medialis \\
\hline Subject 1 & NS & 0.95 & NS & NS \\
\hline Subject 2 & NS & 0.95 & NS & NS \\
\hline
\end{tabular}




\begin{tabular}{|l|l|l|l|l|}
\hline Subject 3 & NS & NS & NS & NS \\
\hline Subject 4 & NS & NS & NS & NS \\
\hline Subject 5 & NS & NS & NS & NS \\
\hline Subject 6 & NS & NS & NS & NS \\
\hline Subject 7 & NS & NS & NS & 0.99 \\
\hline Mean & NS & $\mathbf{0 . 8 8}$ & NS & NS \\
\hline
\end{tabular}

Table 2 reports the correlation between the recorded and computed trends for each subject. In most cases, no significant correlations were found at individual level. On the contrary, a significant correlation between the averaged recorded and simulated activation of the muscles was found for the triceps.

Table 3: Mean $\pm S D$ ranges of motion (SF : shoulder flexion/extension, $S A$ : Shoulder Abduction/adduction, $S I$ : Shoulder internal/external rotation, EF : elbow flexion/extension, WF : Wrist flexion/extension, WT : wrist twist (elbow pronation/supination)), cutting task durations (DUR) and resultant cutting forces $\left(F_{\text {RES }}\right)$ with regard to the workbench height and the cutting direction.

\begin{tabular}{|c|c|c|c|c|c|c|c|c|}
\hline & \multicolumn{2}{|l|}{$0 \mathrm{~cm}$} & \multicolumn{2}{|l|}{$10 \mathrm{~cm}$} & \multicolumn{2}{|l|}{$20 \mathrm{~cm}$} & \multicolumn{2}{|l|}{$30 \mathrm{~cm}$} \\
\hline & direction1 & direction2 & direction1 & direction2 & direction1 & direction 2 & direction 1 & direction2 \\
\hline $\operatorname{SF}\left({ }^{\circ}\right)$ & $20.1 \pm 10.1$ & $15.2 \pm 7.0$ & $28.9 \pm 6.8$ & $19.4 \pm 16.7$ & $49.5 \pm 48.5$ & $35.5 \pm 31.8$ & $40.1 \pm 33.3$ & $29.1 \pm 36.7$ \\
\hline SA $\left({ }^{\circ}\right)$ & $10.2 \pm 3.7$ & $10.7 \pm 5.0$ & $8.6 \pm 5.2$ & $9.3 \pm 4.5$ & $9.9 \pm 4.4$ & $9.3 \pm 3.6$ & $12.6 \pm 5.0$ & $7.8 \pm 3.1$ \\
\hline SI $\left({ }^{\circ}\right)$ & $24.4 \pm 11.7$ & $19.1 \pm 6.3$ & $35.1 \pm 12.8$ & $24.8 \pm 16.4$ & $52.0 \pm 48.8$ & $37.6 \pm 35.7$ & $40.6 \pm 30.3$ & $33.8 \pm 35.5$ \\
\hline $\mathrm{EF}\left({ }^{\circ}\right)$ & $8.5 \pm 3.4$ & $5.9 \pm 2.4$ & $7.1 \pm 3.1$ & $7.3 \pm 1.5$ & $8.8 \pm 3.1$ & $7.4 \pm 3.0$ & $12.2 \pm 4.9$ & $9.0 \pm 4.8$ \\
\hline WF $\left({ }^{\circ}\right)$ & $14.4 \pm 2.8$ & $12.9 \pm 3.3$ & $16.1 \pm 5.1$ & $13.9 \pm 3.8$ & $13.9 \pm 4.7$ & $13.7 \pm 4.9$ & $16.2 \pm 4.7$ & $13.9 \pm 5.2$ \\
\hline DUR (s) & $1.2 \pm 0.4$ & $1.2 \pm 0.5$ & $1.4 \pm 0.5$ & $1.4 \pm 0.6$ & $1.3 \pm 0.6$ & $1.3 \pm 0.6$ & $1.3 \pm 0.5$ & $1.2 \pm 0.5$ \\
\hline $\mathrm{F}_{\mathrm{RES}}(\mathrm{N})$ & $33.4 \pm 8.7$ & $36.8 \pm 12.3$ & $35.5 \pm 10.5$ & $35.5 \pm 8.7$ & $40.5 \pm 10.9$ & $3.8 \pm 12.5$ & $42.7 \pm 13.1$ & $38.6 \pm 12.3$ \\
\hline
\end{tabular}

\subsection{Kinematics and resultant cutting force}

Individual and average values are summarized in table 3. The range of motion of elbow flexion, shoulder abduction, and wrist flexion was small, approx. $4-8 \%$ of the joint capabilities whereas the shoulder internal/external rotation and shoulder flexion were performed at approx. $7-30 \%$ of the joint capabilities. The standard deviations were large underlining the notable differing of motions from one subject to another especially for shoulder rotation and flexion. Similarly, the cutting task duration and the resultant cutting force varied from 0.6 to $2.5 \mathrm{~s}$ and 18 to $67 \mathrm{~N}$, respectively. Workbench height and cutting direction did neither significantly influence the ranges of motion, the resultant cutting force, nor the cutting task duration.

\section{Discussion}

This study investigated the capacity of a musculoskeletal model to predict the relative muscle activation changes as a function of workbench height and cutting direction in a simulated meat cutting task. The correlation coefficients found for recorded and computed muscle activation were similar while the trend analysis depicted some differences. The results indicated that the cutting movements involving arm flexion (direction 1 ) were preferable to the movement requiring internal arm rotation and abduction (direction 2 ). Furthermore, they indicated that the optimal bench height 
should be between 20 and $30 \mathrm{~cm}$ below the elbow height. This confirms that musculoskeletal models can be helpful for the design or re-design of a workstation involving repetitive arm movement.

\subsection{Simulating a work task in laboratory conditions}

The task described here is not a carbon copy of a real meat cutting task, but it involves some of the known physical risk factors such as repetitive arm movement, strenuous, relatively short work cycle duration, and static posture (Sommerich et al., 1993; Bernard, 1997). The task was performed without time and force constraint, and this resulted in inter-subject differences in the joint ranges of motion, cutting task duration and resultant cutting forces (table 3). The simulation of a work task in laboratory conditions enables a practical control of factors affecting the motor behavior like work organization (e.g. productivity requirements), incentive systems, the physical environment, and some psychosocial factors (e.g. perceived time pressure). The present study was designed to control some factors like the static and dynamic phases of the cutting task as well as the order of the task (cutting events). On the contrary, the workspaces as well as the ROM were not constrained to simulate a more real slaughterhouse task. The biomechanical assessments (motion capture, reaction forces, and $E M G$ ) in laboratory conditions enable the use of advanced equipment ensuring high quality recordings as noise sources (electrical, light, and temperature) are controlled (Madeleine et al. 2011). All in all, our study was intended to serve as a basis for investigating the pros and cons of a musculoskeletal model in relation to slaughterhouse work.

Moreover, it should be mentioned that the population size was small, but sufficient to generate new important data concerning the capacity of musculoskeletal modeling to predict relative muscle activation changes in relation to changes in the workbench height and the movement direction.

\subsection{Musculoskeletal simulation and surface electromyography}

The musculoskeletal models have gained in popularity in the modern era of information technology. In ergonomics, Garg and Kappellusch (2009) have summarized the possibility of 2D and 3D biomechanical models for the prevention of WMSD. The AnyBody modeling system is a practical simulation tool that can be used for example during product design and ergonomics interventions. The use of advanced 3D biomechanical models is not cost free, i.e. the recordings and processing of the motion capture and the reaction forces are demanding. Especially, this is the case when the model has to be verified and validated (Lund et al., 2012). However, the approach has also important advantages such as a complete set of muscle forces, joint angles, and joint torques is obtained. One should note that the sole use of EMG recordings in occupational settings only provides information on the level and duration of muscular activation of superficial muscles. Moreover, electrical noise can alter the quality of EMG especially in real conditions (Madeleine et al., 2011).

The musculoskeletal model predicted with precision the trends of the prime movers in average, but failed at predicting individual trends. A possible explanation is that all participants were inexperienced and not instructed to perform the task in a very well-defined way. This resulted in a relatively large standard deviation found in the recorded activation of the muscles and kinematics parameters. As defined in Mathiassen (2006) and Madeleine et al. (2008a), cycle-to-cycle variability can thus explain issues relative to individual trend predictions. If the antagonistic behavior of muscles is not accurately estimated, this will, therefore, result in an underestimation of the synergistic forces considering that the net joint torque remains the same. In other terms, a muscle force estimation algorithm based on net joint torques cannot predict accurately the muscle forces, especially when the subjects are presenting a variable motor pattern. The motor control strategies are affected by the level of experience in meat cutting tasks (Madeleine and Madsen, 2009; Madeleine et al., 2008b). A higher level of experience is reported to lead to a reduction of co-contraction levels (Gribble et al., 2003). The simulation is based on the minimization of the sum of the squared muscle activation of the muscles and does not take different levels of co-contraction into consideration (Praagman et al., 
2010). It is thus conceivable that a lower extent of motor variability could lead to better individual trend predictions in accordance with the force-sharing algorithm definition.

Further, the musculoskeletal model uses a standard scaling algorithm (Andersen et al., 2010) that does not incorporate the differences in force-sharing patterns (Praagman et al., 2010). Discrepancies in isometric maximal muscle forces (Murray et al., 2000) and variations in joint axes and centers can lead to different results of the optimization-based force sharing algorithm (Pierce and Li, 2005). The choice of simple muscle models not taking force-length and force-velocity relationships into account can also result in quantitative errors (Zajac, 1989). Issues related to co-contraction and scaling of the musculoskeletal model are warranted to be addressed in future development of musculoskeletal models.

\subsection{Prediction of muscle activation in relation to workbench height and cutting direction}

The assessment of muscle activation showed that the computed activation of the muscles was estimated more accurately for the muscles involved in the cutting force generation, i.e. deltoideus medialis and triceps, than for the muscles involved in joint stabilization, i.e. biceps and deltoideus anterior. The low correlation coefficients obtained for muscles involved in joint stabilization can be directly explained by the method used to estimate muscle forces (see part 4.2). The stabilizer muscles mostly co-contracted during the investigated task as the subjects had to maintain the knife in the slots. In line with previous studies (Gribble et al., 2003; Osu et al., 2004), co-contraction increases the joint stability and minimizes the perturbing effects of forces arising from limb movement (Osu and Gomi, 1999). More specifically, co-contraction of the biceps and deltoideus anterior muscles played an antagonistic role with respect to the resulting torque of the elbow (producing the cutting force). In such cases, the muscle activation is poorly estimated when using a classical inverse dynamic approach such as the one applied here (Ait-Haddou et al., 2000; Pontonnier and Dumont, 2010). During static postural phases, the biceps contributes to carrying the load of the knife and becomes synergistic. This was why the global correlation coefficient was better than the cutting task correlation coefficient.

Two different cutting directions were investigated in the present study in line with observations from real slaughterhouse work (Madeleine et al., 1999). These different cutting directions consisted of arm flexion (direction 1) and a combination of internal arm rotation and abduction (direction 2). The cutting direction involving arm flexion was found to require lower levels of muscle activation than the cutting direction requiring both internal arm rotation and abduction. This is in agreement with our previous study (Pontonnier et al., 2012) and mostly in line with studies investigating the risk of shoulder disorders in relation to posture (Ohlsson et al., 1995; Punnett et al., 2000). On the contrary, no significant influence of the cutting directions was found on the range of motion, the cutting duration, and the resultant cutting force. This can be explained by the size of the studied population (see 4.1). The lower level of muscular activation found while simulating meat cutting involving arm flexion is most likely of importance. The applied load is one of the well-known physical risk factors for the development of WMSD (Winkel and Westgaard, 1992; Sjøgaard et al., 1995). Thus, the cutting tasks involving arm flexion should be preferred to task requiring both internal arm rotation and abduction.

The muscle activation was also measured and predicted as a function of the workbench height. This type of information is important for practicing ergonomists as it provides an objective quantification of physical load (Garg and Kapellusch, 2000). The predicted activation levels indicated that the optimal height was $30 \mathrm{~cm}$ below the elbow height while the measured ones showed that the optimal height was $-20 \mathrm{~cm}$ in line with Pontonnier et al. (2012). The predicted muscle loads point towards a load transfer from triceps to deltoideus medialis when the workbench height decreased. This implies that the distribution of the muscular activation is changing as a function of workbench height during a simulated meat cutting task. Similar to the cutting directions, there was no significant effect of the workbench height on ranges of motion, cutting duration, or resultant cutting force. Our findings on 
EMG trends indicate that the optimal height in terms of muscular load should be between 20 and 30 $\mathrm{cm}$ below elbow height. This is in line with the general recommendations stating that heavy work should be performed below elbow height (McCormik and Sanders, 1987). However, the optimal height may be challenged by the fact that precision of the cuts is also a very important skill among butchers.

\section{Conclusion}

In conclusion, this study demonstrated that a musculoskeletal model can be useful to obtain a qualitative overview of the force sharing patterns involved in the task even though quantitative estimation of muscle loads remains challenging. Cutting movements involving arm flexion are preferable in terms of biomechanical loads to movement requiring internal arm rotation and abduction. The optimal bench height for the meat cutting task requiring force of approx. $50 \mathrm{~N}$ should be between $20-30 \mathrm{~cm}$ lower than the worker's elbow height. All in all, the present findings confirm that musculoskeletal models can be used to assess musculoskeletal load during the redesign of the work task aiming at reducing physical risk factors for WMSD.

\section{Acknowledgements}

Det Obelske Familiefond supported this study financially. 


\section{References}

Burden, A., 2010. How should we normalize electromyograms obtained from healthy participants? What we have learned from over 25 years of research. J Electromyogr Kinesiol. 20 (6), 1023-1035.

Ait-Haddou, R., Binding, P., Herzog, W., 2000. Theoretical considerations on cocontraction of sets of agonistic and antagonistic muscles. J Biomech. 33 (9), $1105-1111$.

Andersen, M. S., Benoit, D. L., Damsgaard, M., Ramsey, D. K., Rasmussen, J., 2010. Do kinematic models reduce the effects of soft tissue artefacts in skin marker-based motion analysis? an in vivo study of knee kinematics. J Biomech. 43 (2), $268-273$.

Armstrong, T. J., Buckle, P., Fine, L. J., Hagberg, M., Jonsson, B. , Kilbom, A. , Kuorinka, I. A., Silverstein, B. A., Sjogaard, G., Viikari-Juntura, E. R., 1993. A conceptual model for work-related neck and upper-limb musculoskeletal disorders. Scand J Work Environ Health. 19, 73-84.

Bernard, B. P., 1997. Musculoskeletal disorders and workplace factors : a critical review of epidemiologic evidence for work-related musculoskeletal disorders of the neck, upper extremity and low back. Cincinnati, OH: US Department of Health and Human Services.

Buchanan, T. S., Loyd, D. G., Manal, K., Besier, T. F., 2004. Neuromusculoskeletal modeling : Estimation of muscle forces and joints moments and movements from measurements of neural command. J Appl Biomech. 20, 367-395.

Cavanagh, P., Komi, P., 1979. Electromechanical delay in human skeletal muscle under concentric and eccentric contractions. Eur J Appl Physiol Occup Physiol. 42, 159-163.

Damsgaard, M., Rasmussen, J., Christensen, S. T., Surma, E., de Zee, M., 2006. Analysis of musculoskeletal systems in the anybody modeling system. Simul Model Pract Th. 14, 1100-1111.

de Zee, M., Dalstra, M., Cattaneo, P. M., Rasmussen, J., Svensson, P., Melsen, B., 2007. Validation of a musculo-skeletal model of the mandible and its application to mandibular distraction osteogenesis. J Biomech. 40 (6), $1192-1201$.

Erdemir, A., McLean, S., Herzog, W., van den Bogert, A. J., 2007. Model-based estimation of muscle forces exerted during movements. Clin Biomech (Bristol, Avon). 22 (2), 131 - 154.

Eurofound, 2010. Change over time - first findings from the fifth European working conditions survey. European Foundation for the Improvement of Living and Working Conditions.

Forster, E., Simon, U., Augat, P., Claes, L., 2004. Extension of a state-of-the-art optimization criterion to predict co-contraction. J Biomech. 37 (4), 577 - 581.

Garg, A., Kapellusch, J. M., 2009. Applications of biomechanics for prevention of work-related musculoskeletal disorders. Ergonomics. 52(1), 36-59.

Gribble, P. L., Mullin, L. I., Cothros, N., Mattar, A., 2003. Role of cocontraction in arm movement accuracy. J Neurophysiol. 89(5), 2396-2405.

Juul-Kristensen, B., Fallentin, N., Hansson, G. A., Madeleine, P., Andersen, J. H., Ekdahl, C., 2002. Physical workload during manual and mechanical deboning of poultry. Int J Ind Ergon. 29, 107-115.

Lund, M. E., de Zee, M., Andersen, M. S., Rasmussen, J., 2012. On validation of multibody musculoskeletal models. Eng Med. 226, 82-94. 
Madeleine, P. Farina, D., 2008. Time to task failure in shoulder elevation is associated to increase in amplitude and to spatial heterogeneity of upper trapezius mechanomyographic signals. Eur. J. Appl. Physiol. 102, 325-333.

Madeleine, P., Madsen, T. M. T., 2009. Changes in the amount and structure of motor variability during a deboning process are associated with work experience and neck-shoulder discomfort. Appl Ergon. 40 (5), $887-894$.

Madeleine, P., Lundager, B., Voigt, M., Arendt-Nielsen, L., 1999. Shoulder muscle coordination under chronic and experimental neck shoulder pain: An occupational pain study. Eur. J. Appl. Physiol. 79, $127-140$.

Madeleine, P., Mathiassen, S. E., Arendt-Nielsen, L., 2008. Change in the degree of motor variability associated with experimental and chronic neck-shoulder pain during a standardized repetitive arm movement. Exp Brain Res. 185, 689-698.

Madeleine, P., Samani, A., de Zee, M., Kersting, U.G., 2011. Biomechanical assessments in sports and ergonomics. In Theoretical Biomechanics / Book, Ed, Klika V. InTech, Vienna, Austria.

Madeleine, P., Voigt, M., Mathiassen, S. E., 2008. The size of cycle-to-cycle variability in biomechanical exposure among butchers performing a standardised cutting task. Ergonomics, 51(7), 1078-1095.

Mathiassen, S. E., 2006. Diversity and variation in biomechanical exposure: What is it, and why would we like to know? Appl Ergon. 37 (4), $419-427$.

McCormik, E. J., Sanders, M. S., 1987. Human Factors in engineering and design, New York: McGrawHill.

McGorry, R. W., 2001. A system for the measurement of grip forces and applied moments during hand tool use. Appl Ergon. 32, 271-279.

McGorry, R. W., Dowd, P. C., Dempsey, P. G., 2003. Cutting moments and grip forces in meat cutting operations and the effect of knife sharpness. Appl Ergon. 34, 375-382.

Murray, W. M., Buchanan, T. S., Delp, S. L., 2000. The isometric functional capacity of muscles that cross the elbow. J Biomech. 33, 943-952.

Ohlsson, K., Attewell, R.G., Pålsson, B., Karlsson, B., Balogh, I., Johnsson, B., Ahlm, A., Skerfving, S. (1995). Repetitive industrial work and neck and upper limb disorders in females. Am J Ind Med. 27, 731-747.

OSHA. Lighten the load, 2007. Magazine of the European Agency for Safety and Health at Work.

Osu, R., Gomi, H., 1999. Multijoint muscle regulation mechanisms examined by measured human arm stiffness and emg signals. J Neurophysiol. 81 (4), 1458-1468.

Osu, R., Kamimura, N., Iwasaki, H., Nakano, E., Harris, C. M., Wada, Y., Kawato, M., 2004. Optimal impedance control for task achievement in the presence of signal-dependent noise. J Neurophysiol. 92 (2), 1199-1215.

Pierce, J.E., Li, G., 2005. Muscle forces predicted using optimization methods are coordinate system dependent. J Biomech. 38 (4), $695-702$.

Pontonnier, C., Dumont, G., 2010. From motion capture to muscle forces in the human elbow aimed at improving the ergonomics of workstations. Virtual Phys Prototyp. 5, 113-122. 
Pontonnier, C., de Zee, M., Samani, A., Dumont, G., Madeleine, P., 2011. Meat Cutting Tasks Analysis using 3D Instrumented Knife and Motion Capture. IFMBE Proceedings. 34, 144-147.

Pontonnier, C., de Zee, M., Samani, A., Dumont, G., Madeleine, P., 2012. Cutting force and emg recording for ergonomics assessment of meat cutting tasks : influence of the workbench height and the cutting direction on muscle activation levels. In ASME 2012 11th Biennial Conference On Engineering Systems Design And Analysis (ESDA2012).

Praagman, M., Chadwick, E. K. J., van der Helm, F. C. T., Veeger, H. E. J., 2010. The effect of elbow angle and external moment on load sharing of elbow muscles .J Electromyogr Kinesiol. 20, 912-922.

Punnett, L., Fine, L.J., Keyserling, W.M., Herrin, G.D., Chaffin, D.B., 2000. Shoulder disorders and postural stress in automobile assembly work. Scand J Work Environ Health.26, 283-291.

De Zee, M., Rasmussen, J. 2010. Computational investigation of two interventions for neck and upper extremity pain in office workers. IFMBE Proceedings. 31, 64-66.

SENIAM, 1999. The State of the Art on Signal Processing Methods for Surface ElectroMyoGraphy. Roessingh Research and Development, Enschede, the Netherlands.

Sjøgaard, G., Sejersted, O. M., Winkel, J., Smolander, J., Jørgensen, K., Westgaard, R.H., 1995. Exposure assessment and mechanisms of pathogenesis in work-related musculoskeletal disorders: significant aspects in the documentation of risk factors. Work and health: Scientific basis of progress in the working environment, European Comission DG V, Luxembourg, 75-87.

Sommerich, C. M., McGlothin, J., Marras, W. S., 1993. Occupational risk factors associated with soft tissue disorders of the shoulder: a review of recent investigations in the literature. Ergonomics. 36, 697-717.

Staudenmann, D., Kingma, I., Stegeman, D. F., van Dieën, J. H., 2005. Towards optimal multi-channel emg electrode configurations in muscle force estimation: a high density emg study. J Electromyogr Kinesiol. 15 (1), $1-11$.

Staudenmann, D., Roeleveld, K., Stegeman, D. F., van Dieën, J. H., 2010. Methodological aspects of semg recordings for force estimation - a tutorial and review. J Electromyogr Kinesiol. 20 (3), 375 387.

Van Der Helm, F. C. T., 1994. A finite element musculoskeletal model of the shoulder mechanism. J Biomech. 27, 593-633.

van Dieën, J. H., Thissen, C., van de Ven, A., Toussaint, H., 1991. The electro-mechanical delay of the erector spinae muscle: influence of rate of force development, fatigue and electrode location. Eur $\mathrm{J}$ Appl Physiol Occup Physiol. 63, 216-222.

Vos, E. J., Harlaar, J., van Ingen Schenau, G. J., 1991. Electromechanical delay during knee extensor contractions. Med Sci Sports Exerc. 23(10), 1187-1193.

Weidlich, D., Cser, L., Polzin, T., Cristiano, D., Zickner, H., 2009. Virtual reality approaches for immersive design. IJIDeM. 3, 103-108.

Winkel, J., Westgaard, R., 1992. Occupational and individual risk factors for shoulder-neck complaints: Part ii-the scientific basis (literature review) for the guide. Int J Ind Ergon. 10, 85-104.

Zajac, F.E., 1989. Muscle and tendon : properties, models, scaling and application to biomechanics and motor control. Crit Rev Biomed Eng. 17(4), 359-411. 